\title{
Two major ruminant acute phase proteins, haptoglobin and serum amyloid $A$, as serum biomarkers during active sheep scab infestation
}

\author{
Beth Wells ${ }^{1 *}$, Giles T Innocent ${ }^{2}$, Peter D Eckersall ${ }^{3}$, Eilidh McCulloch ${ }^{4}$, Alasdair J Nisbet ${ }^{1}$ and Stewart TG Burgess ${ }^{1}$
}

\begin{abstract}
Two ruminant acute phase proteins (APPs), haptoglobin ( $\mathrm{Hp}$ ) and serum amyloid A (SAA), were evaluated as serum biomarkers (BMs) for sheep scab-a highly contagious ectoparasitic disease caused by the mite Psoroptes ovis, which is a major welfare and production threat worldwide. The levels of both APPs increased in serum following experimental infestation of sheep with $P$. ovis, becoming statistically significantly elevated from pre-infestation levels at 4 weeks post-infestation. Following successful treatment of infested sheep with an endectocide, Hp and SAA serum levels declined rapidly, with half lives of less than 3 days. In contrast, serum IgG levels which specifically bound the P. ovis-derived diagnostic antigen Pso o 2 had a half-life of 56 days. Taking into account pre-infestation serum levels, rapidity of response to infestation and test sensitivity at the estimated optimum cut-off values, SAA was the more discriminatory marker. These studies illustrated the potential of SAA and Hp to indicate current sheep scab infestation status and to augment the existing Pso o 2 serological assay to give disease-specific indications of both infestation and successful treatment.
\end{abstract}

\section{Introduction}

Sheep scab, caused by the infestation of sheep skin with the highly contagious mite Psoroptes ovis, affects the productivity and welfare of sheep as it produces intensely pruritic lesions and wool loss. These factors, along with the high cost of treatment, mean this disease has significant economic implications for the sheep industries of affected countries [1]. In the UK, the incidence of the disease has increased to a level that it is now endemic [2] with the national annual prevalence estimated at $>7000$ outbreaks [3]. This has resulted in the disease being made notifiable in a number of countries (e.g. in Scotland through the Sheep Scab (Scotland) Order 2010 [4]), which has renewed the focus on accurate diagnosis for effective control. Accurate diagnosis can be challenging - clinical signs in $P$. ovis infested sheep may be minimal during early infestation (up to several months in the field) and easily missed but these animals represent a source of infestation [5]. In such a

\footnotetext{
* Correspondence: beth.wells@moredun.ac.uk

'Moredun Research Institute, Pentlands Science Park, Midlothian EH26 OPZ, UK Full list of author information is available at the end of the article
}

situation, mites can be difficult to locate and the sensitivity of the microscopic detection of mites in skin scrapings from these animals can be as low as 18\% [6]. In addition to $P$. ovis, other ectoparasites such as ticks and lice can induce skin reactions which may appear similar to those seen in early infestation with $P$. ovis $[7,8]$ and dual infestations with more than one ectoparasite are common. The difficulties highlighted above in the control and diagnosis of sheep scab illustrates the requirement for a sensitive and specific test which would indicate early infestation and also current disease status.

Several immunoassays have been developed to detect serum antibodies with specific reactivity to $P$. ovis antigens from infested sheep [9-11]. Recently we have successfully developed a diagnostic test which detects the host's antibody response to the $P$. ovis antigen Pso o 2 . This test is highly specific and sensitive and can detect serum antibodies to Pso o 2 as early as two weeks postinfestation [12]. However, due to antibody persistence of several months in the serum, the test cannot distinguish between currently-infested and recently-(successfully) treated sheep [13]. This is an important factor in any control or eradication regime where demonstration of 
freedom from infestation following treatment forms part of legislation on movement restrictions etc. Therefore, an improved diagnostic test for sheep scab should combine the pathogen specificity and early indication of infestation (as provided by the Pso o 2 ELISA [12]) with a biomarker (BM) element to differentiate between successfully-treated animals and those with active disease.

Ideally, BMs should circulate at low levels in the serum of healthy individuals, increase in titre rapidly following the onset of disease and return to pre-infestation levels soon after successful treatment or disease resolution [14]. As this describes the behaviour of inflammatory proteins and, as sheep scab produces an acute inflammatory response within hours of infestation [15], we investigated two ruminant acute phase proteins (APPs)-haptoglobin (Hp) and serum amyloid A (SAA)-as BMs for sheep scab. Ruminants are unique in that $\mathrm{Hp}$ is a major APP [16] and in sheep, as for cattle, APP production continues in chronic as well as acute infections, with roles in tissue repair. In cattle, $\mathrm{Hp}$ is synthesised only in response to inflammation [17] and Hp levels have been used extensively to monitor inflammatory disease [18] but to date there has been very little work on the acute phase response in sheep [16].

This study investigates qualitative and quantitative serum levels of $\mathrm{Hp}$ and SAA in sheep during experimental infestations with $P$. ovis and the effects of successful treatment on these APPs; reports Hp and SAA levels during other common conditions and diseases of sheep and in sheep diagnosed as clinically positive during a natural outbreak of sheep scab.

\section{Materials and methods}

\section{Samples used in APP evaluation} Time course trial

To supply sera for initial immunoblot analyses, blood was sampled from each of 6 Scotch Mule mixed sex 1-2 year old sheep, by venous extraction, prior to infestation with $P$. ovis. Following infestation on the withers with approximately 50 mites, blood was sampled weekly from each animal for 6 weeks and sera were prepared by centrifuging whole blood at $900 \mathrm{~g}$ for $10 \mathrm{~min}$. Sera were then stored at $-20{ }^{\circ} \mathrm{C}$ until use. This part of the study was termed the "time-course trial" (TCT).

\section{Primary infestation and treatment trial}

For the quantitative analysis of $\mathrm{Hp}$ and SAA, sera were obtained from 12 P. ovis-naïve, Scotch Mule, mixed sex, 1-2 year old sheep infested with $P$. ovis mites as described above. Blood samples were acquired before exposure and then weekly over a 6 week period postinfestation. At 6 weeks post-infestation, all twelve sheep were treated with an injectable endectocide (Dectomax, Pfizer Animal Health) by intramuscular injection at a dose of $1 \mathrm{~mL}$ per $33 \mathrm{~kg}$ bodyweight and blood was then sampled twice weekly for 2.5 weeks; then at 4, 9 and 14 weeks post-treatment. This part of the study was termed the "primary infestation and treatment" (PIT) trial.

\section{Secondary infestation and treatment trial}

Fourteen weeks post-treatment the sheep were re-infested with $P$. ovis and blood samples were taken at 24 hours post-infestation then weekly for a further 6 weeks. On week 6 , sheep were treated with endectocide again, as described above, and blood was then sampled twice weekly for a further 2.5 weeks. This part of the study was termed the "secondary infestation and treatment" (SIT) trial. Sera were prepared from each of the blood samples by centrifuging whole blood at $900 \mathrm{~g}$ then sera were stored at $-20{ }^{\circ} \mathrm{C}$ until use. Lesion areas were measured at the time of each blood sampling post-infestation until 2 weeks post-treatment by measuring the length and width of the main lesion on each sheep and recording the result as an average mean lesion area $\left(\mathrm{cm}^{2}\right) \pm$ SEM.

\section{Field acquired infestations}

In addition to these sera from experimental infestations, sera were also acquired from sheep with field-acquired sheep scab infestations $(n=12)$. Sera were available for these animals pre-infestation, at point of clinical diagnosis and two months post-treatment [13].

\section{Other common infections}

To test the specificity of the $\mathrm{Hp}$ and SAA response, the samples listed in Table 1 were used to analyse $\mathrm{Hp}$ and

Table 1 Sera samples used to test APP responses during common infections and conditions of sheep

\begin{tabular}{lc}
\hline Infection / condition & Number of samples \\
\hline Early gestation $^{\mathrm{a}}$ & 10 \\
Late gestation $^{\mathrm{b}}$ & 10 \\
Gastro-intestinal nematodes $^{c}$ & 6 \\
Liver Fluke (Fasciola hepatica) $^{\mathrm{d}}$ & 6 \\
Sucking lice (Linognathus spp.) $^{\mathrm{e}}$ & 4 \\
Chewing lice (Bovicola ovis) $^{\mathrm{e}}$ & 4 \\
Orf $^{\mathrm{f}}$ & 6 \\
Johnes disease $^{\mathrm{g}}$ & 6
\end{tabular}

${ }^{a}$ Early gestation was within 1 week of conception and ${ }^{b}$ late gestation was 1 week prior to expected lambing date (samples from Moredun Research Institute (MRI)). 'Samples from sheep with field-acquired gastro-intestinal nematode (GIN) infections were from Firth Mains Farm, UK (MRI); species included Teladorsagia circumcincta and Nematodirus battus. 'Samples were taken from UK sheep with field-acquired infections of liver fluke (samples provide by Dr H McDougall, MRI); ${ }^{\mathrm{e}}$ samples from lice infected but $P$. ovis-uninfested sheep (provided by Prof. N. Sargison, R(D)SVS, UK; ${ }^{{ }^{\prime}}$ samples from sheep infected experimentally with orf virus or ${ }^{9}$ Mycobacterium avium subspecies paratuberculosis (MRI). 
SAA levels during other common infections and conditions of sheep.

\section{Qualitative analyses of the APPs: Haptoglobin (Hp) and Serum Amyloid A (SAA)}

Sera from each of 6 sheep in the TCT study were pooled according to time point post-infestation and were then diluted 1:10 with $\mathrm{dH}_{2} \mathrm{O}$. Polyacrylamide gel electrophoresis (SDS-PAGE), under denaturing conditions, was performed using NuPage BisTris 4-12\% polyacrylamide gels (Invitrogen) with MES buffer (Invitrogen) according to the manufacturer's instructions.

Gels were stained in SimplyBlue Safestain (Invitrogen) following the manufacturer's protocol. Bands which increased in intensity through the time course of infestation were excised from the gel and identified using matrix assisted laser desorption ionization (MALDI) at the Moredun Proteomics Facility: Excised bands were destained and subjected to reductive alkylation using DTT and iodoacetamide. Gel pieces were digested overnight at $37{ }^{\circ} \mathrm{C}$ in trypsin and digests analysed on an Ultraflex II MALDI-ToF-ToF mass spectrometer (Bruker Daltonics). The masses obtained were used for database searching with the MASCOT search engine using SwissProt and local databases with a $50 \mathrm{ppm}$ mass tolerance window. Significant matches from the Peptide Mass Fingerprint data were confirmed by MS/MS analysis using the search criteria above and an MS/MS tolerance window of $0.5 \mathrm{Da}$.

Immunoblotting was performed using sera from individual sheep with different disease progression profiles in the TCT trial. Sheep 1 had a naturally-resolving lesion, which was at maximum size at week 4 postinfestation, whilst the lesion from Sheep 5 and 6 showed a gradual expansion over the time course of infestation. Sera from these sheep from each time point in the TCT trial were separated by SDS-PAGE as described above and then transferred to a nitrocellulose membrane by electroblotting using the iBlot Western Blotting system (Invitrogen), following manufacturer's instructions. The blot was blocked using 3\% gelatin from cold water fish skin (Aldrich) for $30 \mathrm{~min}$ prior to washing with washing buffer/antibody diluent (10\% PBS; 90\% d $\mathrm{dH}_{2} \mathrm{O}, 29.22 \mathrm{~g}$ $\mathrm{NaCl}$ and $5 \mathrm{~mL}$ Tween 80 (Sigma-Aldrich). The blot was incubated with the following antibodies for $1 \mathrm{~h}$ with washing in between incubations: The primary antibody used for detecting $\mathrm{Hp}$ in the electrophoresed serum samples was a rabbit polyclonal anti-human Hp (Abcam, ab85846) used at a concentration of $1 \mu \mathrm{g} / \mathrm{mL}$. The secondary antibody conjugate used was a swine anti-rabbit IgG HRP conjugate (Dako, P0399) used at a concentration of $0.5 \mu \mathrm{g} / \mathrm{mL}$. Visualisation was by ECL Plus (GE Healthcare) using the ImageQuant system (GE Healthcare). The primary antibody for the detection of SAA was a rabbit polyclonal anti-human recombinant SAA (Abcam, ab59736) used at a concentration of $2 \mu \mathrm{g} / \mathrm{mL}$. The conjugate was a swine anti-rabbit HRP IgG (Dako P0399) used at a concentration of $0.5 \mu \mathrm{g} / \mathrm{mL}$. Band densities were estimated using the Quantity One software 4.6.2 (Bio-Rad).

\section{Quantitative analyses of the AAPs}

Hp concentrations were determined by ReactivLab and Glasgow University using a colorimetric assay (ReactivLab Ltd, Wetherby, UK) based on the method described by Eckersall et al. [19] and modified as described in International Patent Application WO 2012/085497 A1. Validation of this cross species assay for $\mathrm{Hp}$ was described in Crawford et al. [20]. For determination of the concentration of $\mathrm{Hp}$ in ovine serum the assay was further validated. The intra-assay coefficient of variance (CV) was $5.5 \%$ calculated as the mean of CVs of samples $(n=47)$ run in duplicate in one assay, the inter-assay CVs using quality control samples of ovine serum were $6.5 \%$ at a mean $\mathrm{Hp}$ of $0.34 \mathrm{~g} / \mathrm{L}(n=7), 5 \%$ at a mean $\mathrm{Hp}$ of $0.71 \mathrm{~g} / \mathrm{L}(n=7)$ and $11.5 \%$ at a mean $\mathrm{Hp}$ of $1.01 \mathrm{~g} / \mathrm{L}$ $(n=5)$. The limit of detection was $0.02 \mathrm{~g} / \mathrm{L}$ determined as the concentration of $\mathrm{Hp}$ at 3 standard deviations from the mean of a blank sample. Hp concentrations were quantified in sheep sera from the PIT and SIT trials; from the field-acquired $P$. ovis infestations and from sheep with other common conditions (Table 1). The Hp assay was performed on an ABX Pentra 400 analyser (Horiba Medical) using a calibration curve with a top $\mathrm{Hp}$ standard of $1.48 \mathrm{mg} / \mathrm{mL}$ such that samples with $\mathrm{Hp}$ values greater than this were automatically diluted. All samples were tested in duplicate.

For SAA, a commercially-available ELISA kit (TP-802, Tridelta Development Ltd) was used to quantify levels of this APP in sheep sera. The manufacturer's protocol was followed using the same sera samples as described above for Hp. Samples were tested from individuals in duplicate alongside a SAA standard curve and all serum was diluted 1:500 in sample diluent buffer (Tridelta Development Ltd). The upper level of the range of the bovine standards used was $300 \mathrm{ng} / \mathrm{mL}$ therefore all sera samples showing SAA values greater than this were further diluted as required and re-analysed.

\section{Analysis of serum IgG levels to the mite antigen Pso o 2}

An ELISA which measures the levels of Pso o 2-specific IgG in sheep serum [12] was used to allow post-infestation comparisons of Pso o 2-specific IgG synthetic and decay profiles (post-treatment) with those of Hp and SAA. Serum samples from the PIT and SIT experimental trials were used to assess persistence in antigen-specific IgG levels after treatment $[11,13]$. Serum samples were analysed up to, and including, 14 weeks post-treatment. The ELISA was performed as previously described [12] with the 
following modifications: all sera samples were assayed for individual sheep in duplicate. Positive, inter-plate controls were used from sheep $(n=2)$ which had been re-infested with $P$. ovis for 6 weeks following a primary infestation (6 weeks) and effective treatment. Negative controls consisted of the pre-infestation sera from the PIT trial sheep.

\section{Statistical analysis}

Initial statistical analyses of $\mathrm{Hp}$ and SAA levels in the PIT and SIT trials were performed using repeated measures one-way analysis of variance (ANOVA) with a Tukey's post-hoc test and performed in Graph Pad Prism (Version 5.05, GraphPad Software Inc). Further statistical analyses were performed by Biomathematics and Statistics Scotland (BioSS) using the R Statistical Package [21]: Best fit models were identified to describe $\mathrm{Hp}$, SAA and Pso o 2-specific IgG trends (as "normal" or "elevated" compared to pre-infestation levels) over the time course of infestation and post-treatment; half life values were calculated using a generalised linear mixed model to describe the exponential decay of the BMs and Pso o 2-specific IgG and incorporated a random effect of animal on the decay constant, and the initial value. In addition, optimised cut-off values were calculated, where the objective was to obtain as high sensitivity and specificity as possible to keep false results to a minimum. This was achieved using a generalised linear mixed model for binomial data, with a logit link which required the use of the library "Ime4" [22].

\section{Results}

Qualitative analyses of the APPs: Hp and SAA from the TCT trial

Mean lesional areas are shown in Figure 1 and illustrate gradual lesion growth until week 4 post-infestation, followed by rapid expansion between week 4 to week 6 postinfestation. Post-treatment, the lesions resolved slowly and measurement was stopped 2 weeks after treatment due to the nature of lesion healing as they healed and lifted off rather than decreased in size.

The densities of two bands, with approximate molecular masses of 17 and $38 \mathrm{kDa}$, increased through the time course of infestation with $P$. ovis, with maximum abundance at weeks 5 and 6 post-infestation (Figure 2).

MALDI analysis identified both bands as fragments of $\mathrm{Hp}$. As the ovine Hp sequence has not yet been deposited in public databases, the polypeptide with highest homology to the $17 \mathrm{kDa}$ band was Capra ibex $\mathrm{Hp}$ with sequence coverage of $35.7 \%$ and intensity coverage of $79.7 \%$ (mascot score 127). For the $38 \mathrm{kDa}$ sample, the polypeptide with highest homology was Cervus elaphus $\mathrm{Hp}$ with an intensity coverage of $11.3 \%$ and sequence coverage of $14.5 \%$ (mascot score 202).

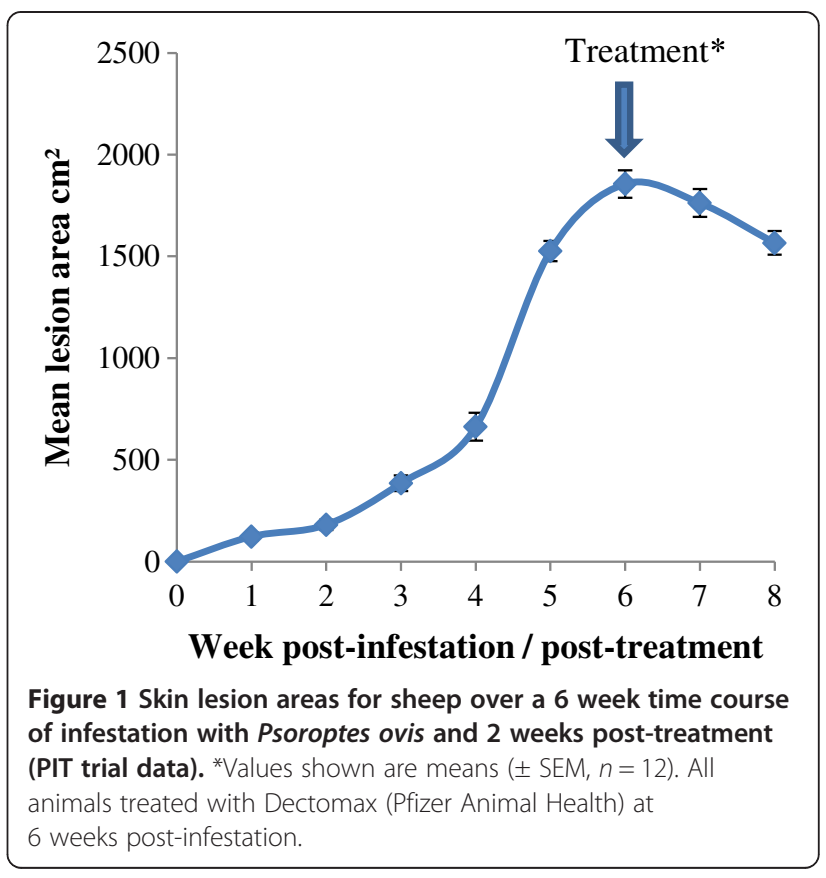

Using immunoblot and densitometry analysis, along with lesion area data from trial TCT, it was demonstrated that, as the lesion size increased, the band density of $\mathrm{Hp}$ (at $45 \mathrm{kDa}$ ) also increased and as the lesion resolved the intensity of the bands decreased (Figure 3). For SAA, the densities of bands obtained in immunoblots, at the estimated

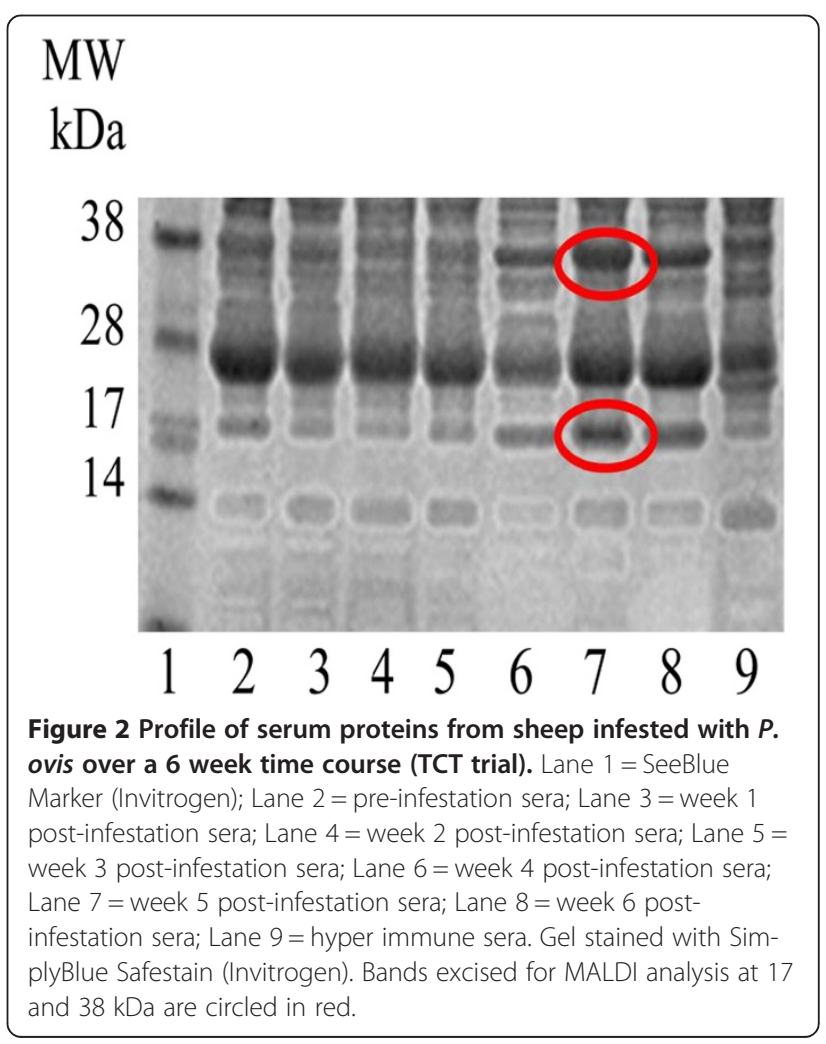




Land density
Figure 3 Relationship between lesion area and serum Hp during a 6 week infestation of sheep with Psoroptes ovis. Panel a: Serum from
a sheep infested with P. ovis in trial TCT where the lesion resolved naturally after 4 weeks (Sheep 1); Panel b: Serum from an infested sheep in the
same trial where the lesion had continued to expand during the 6 week time course (Sheep 5). Immunoblots were probed with a rabbit
polyclonal anti-human Hp antibody (Abcam) and the secondary antibody conjugate used was a swine anti-rabbit lgG HRP (Dako). The image was
visualised by ECL Plus and ImageQuant. Band volumes (at 45 kDa) were quantified by ImageQuant TL software.

MW of SAA (14 kDa), were also measured (Figure 4) and a clear relationship between SAA band density and lesion size was identified, with SAA band density peaking 2 weeks before the lesion size peak in the lesion resolving sheep (Figure 4a).

\section{Quantitative analyses of the APPs}

The mean Hp concentration in sheep serum pre-infestation with $P$. ovis was $0.30 \pm 0.06 \mathrm{mg} / \mathrm{mL}$. There was no statistically significant increase in $\mathrm{Hp}$ levels at the $5 \%$ level during the primary infestation until after 4 weeks post-infestation (Figure 5a). Between weeks 4 and 5 post-infestation, mean $\mathrm{Hp}$ levels increased from $0.83 \pm 0.41 \mathrm{mg} / \mathrm{mL}$ to $3.33 \pm$ $0.89 \mathrm{mg} / \mathrm{mL}(p \leq 0.001)$. Following termination of the primary infestation with endectocide, mean $\mathrm{Hp}$ levels fell significantly $(p \leq 0.001)$ from $3.53 \pm 0.64 \mathrm{mg} / \mathrm{mL}$ at the point of treatment to $1.57 \pm 0.35 \mathrm{mg} / \mathrm{mL}$ one week post-treatment and had returned to pre-infestation levels between 10 and 14 days after treatment. During this PIT phase of the trial, the proportion of animals with "elevated" Hp increased between weeks 4 and 5 post-infestation and all, except one, animal had returned to "normal" by 10 days post-treatment (Table 2). The half life of Hp levels in the serum posttreatment was calculated as 2.3 days [ $95 \%$ confidence interval $(\mathrm{CI})=1.9-2.9]$ and a cut-off of $1.26 \mathrm{mg} / \mathrm{mL}$ was selected, giving an estimated sensitivity of 0.83 and a specificity of 1.0 .

The $\mathrm{Hp}$ profile during the secondary infestation (Figure 5a) showed that serum $\mathrm{Hp}$ levels increased more rapidly following this secondary challenge than during the primary infestation with $P$. ovis. Hp levels had doubled by 24 hours post-infestation in the secondary infestation compared with the much slower increase in levels during the primary infestation. The maximum $\mathrm{Hp}$ concentration measured during the secondary infestation
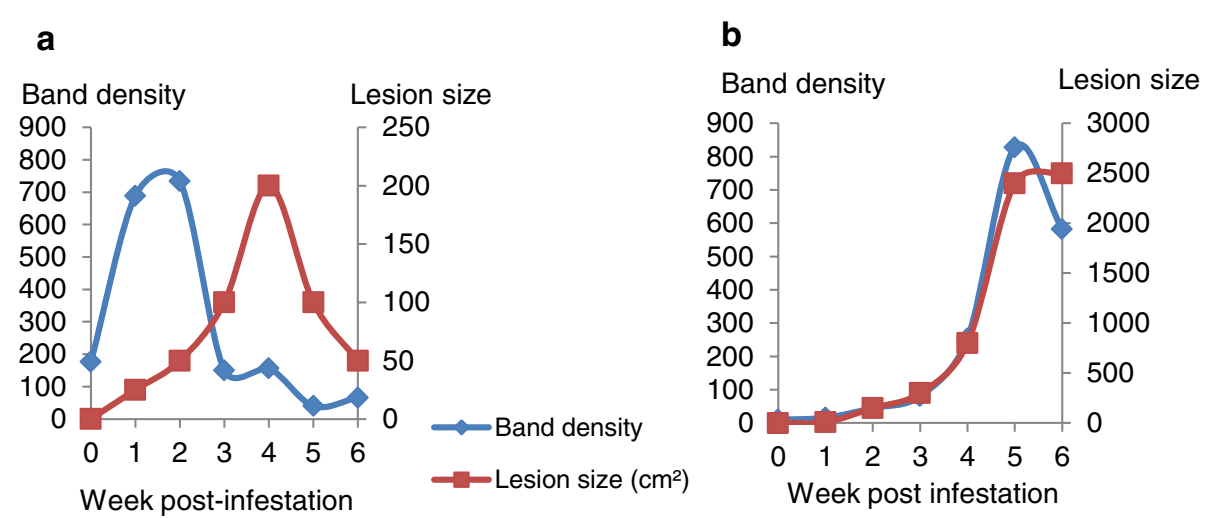

Figure 4 Relationship between lesion area and serum SAA during a 6 week infestation of sheep with Psoroptes ovis. Panel a: Serum from a sheep infested with P. ovis in trial TCT where the lesion resolved naturally after 4 weeks (Sheep 1); Panel $\mathbf{b}$ : Serum from an infested sheep in the same trial where the lesion had continued to expand during the 6 week time course (Sheep 6). The blots were probed with an anti-SAA antibody raised in rabbits against human rSAA (Abcam) and the secondary antibody conjugate was swine anti-rabbit HRP IgG (Dako). The blot was visualised using ECL Plus (GE Healthcare) and ImageQuant. Band volumes (at $14 \mathrm{kDa}$ ) were quantified by ImageQuant TL software. 

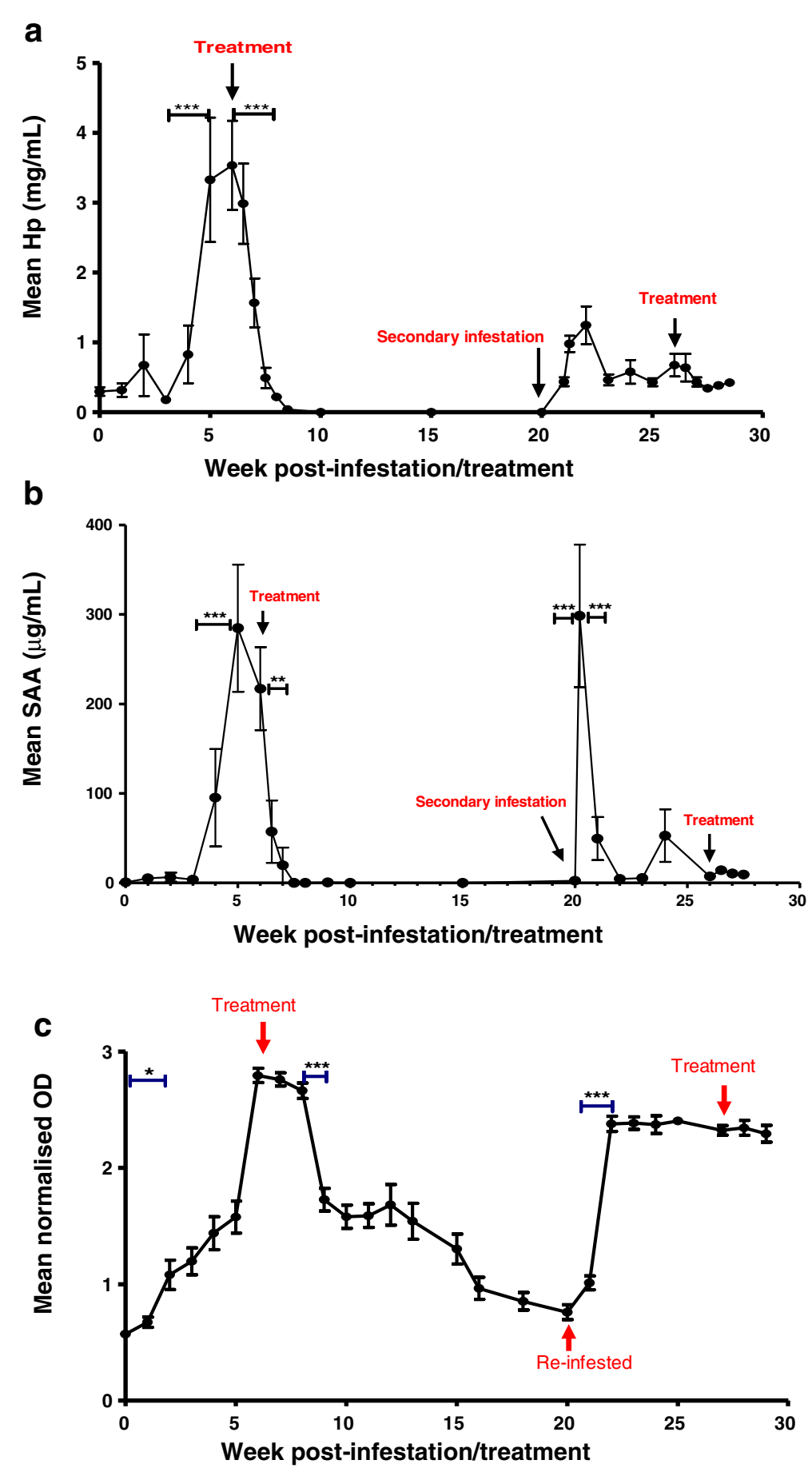

Figure 5 Quantitative analyses of serum acute phase protein and Pso o 2-specific lgG levels during an infestation/treatment/re-infestation model of Psoroptes ovis in sheep. Panel a: Haptoglobin: Sera samples from individual sheep $(n=12)$ in the PIT and SIT trial were analysed in duplicate using a colorimetric assay (ReactivLab). Hp concentrations are shown as mean ( \pm SEM) through the time course of primary infestation (PIT) with P. ovis for 6 weeks, and post-treatment over 14 weeks, followed by secondary infestation (SIT) for a further 6 weeks and post-treatment for 2.5 weeks. Panel b: SAA: Sera samples from individual sheep $(n=12)$ in the PIT and SIT trial were analysed in duplicate using SAA ELISA (Tridelta Development Ltd). SAA concentrations are shown as mean ( \pm SEM) through the time course of primary infestation (PIT) with P. ovis for 6 weeks, and post-treatment over 14 weeks, followed by secondary infestation (SIT) for a further 6 weeks and post-treatment for 2.5 weeks. Panel c: Pso o 2-specific lgG: Sera samples from individual sheep $(n=12)$ in the PIT and SIT trial were analysed in duplicate using an ELISA in which the coating antigen was a recombinant Pso $\circ 2$ (rPso o 2) at $75 \mu \mathrm{g} / \mathrm{mL}$; primary antibody was lgG in the serum samples and the conjugate was a rabbit anti-sheep lgG HRP (Dako) at a dilution of 1:2000. lgG levels are represented as mean normalised $\mathrm{OD}_{450}$ values $( \pm \mathrm{SEM}) .{ }^{*} p \leq 0.05 ;{ }^{* *} p \leq 0.01$ and ${ }^{* *} p \leq 0.001$ as determined by repeated measures one-way ANOVA (Graph Pad Prism). 
Table 2 Distribution of sheep into "normal" and "elevated" groups for serum Hp and SAA concentration across the time course of primary infestation with Psoroptes ovis and treatment with an endectocide (PIT trial)

\begin{tabular}{ccccccccccccc}
\hline Biomarker & Week & $\mathbf{0}$ & $\mathbf{1}$ & $\mathbf{2}$ & $\mathbf{3}$ & $\mathbf{4}$ & $\mathbf{5}$ & $\mathbf{6}^{* *}$ & $\mathbf{6 . 5}$ & $\mathbf{7}$ & $\mathbf{7 . 5}$ & $\mathbf{8}$ \\
\hline $\mathbf{H p}$ & Normal $^{*}$ & 12 & 11 & 11 & 11 & 10 & 4 & 2 & 2 & 7 & 11 & 12 \\
& Elevated & 0 & 1 & 1 & 1 & 2 & 8 & 10 & 10 & 5 & 1 & 0 \\
\multirow{3}{*}{ SAA } & Normal & 12 & 9 & 10 & 11 & 4 & 0 & 0 & 7 & 11 & 12 & 12 \\
& Elevated & 0 & 3 & 2 & 1 & 8 & 12 & 12 & 5 & 1 & 0 & 0
\end{tabular}

* The status of APP levels in sheep sera ( $n=12$ for each time point) was determined by comparison to pre-infestation levels using the $R$ Statistical Package.

** All sheep were treated with an injectable endectocide at week 6 postinfestation. In these analyses the animals were described as being "normal" as in day 0 (pre-infestation) or "elevated". As the results showed a normal distribution, all values above the 0.999 quantile of the distribution were considered to be elevated, giving a $99.9 \%$ specificity and undefined sensitivity.

was at 2 weeks post-infestation (week 22) with a value of $1.25 \pm 0.27 \mathrm{mg} / \mathrm{mL}$. By week 3 in the secondary infestation, $\mathrm{Hp}$ levels had decreased to pre-infestation levels and remained at low levels for the remainder of the trial.

SAA levels pre-infestation were $0.82 \pm 0.53 \mu \mathrm{g} / \mathrm{mL}$ and remained at this level over the first 3 weeks of primary infestation (PIT, Figure 5b). By week 4 post-infestation, SAA levels had increased to $95.24 \pm 54.4 \mu \mathrm{g} / \mathrm{mL}$ and thereafter peaked at week $5(284.75 \pm 71.144 \mu \mathrm{g} / \mathrm{mL})$. The increase in serum SAA levels between weeks 3 and 5 post-infestation was highly statistically significant $(p \leq$ 0.001). Post-treatment, serum SAA levels fell within 3 days of treatment to $57.28 \pm 34.75 \mu \mathrm{g} / \mathrm{mL}(p \leq 0.05)$ and continued to decrease reaching pre-infestation levels within 10 days post-treatment. The proportion of animals showing elevated serum SAA increased between weeks 4 and 5 post-infestation and all except one animal had returned to normal by 10 days posttreatment (Table 2). The half life of SAA in the serum post-treatment was estimated at 0.84 days $(95 \% \mathrm{CI}=0.73$ 0.99 ) and a cut-off value of $29.5 \mu \mathrm{g} / \mathrm{mL}$ was selected to identify "elevated" animals above that point or "normal" animals below it. This cut-off value provided an estimated assay sensitivity of 1.0 and a specificity of 1.0.

The secondary infestation was characterised by a rapid increase in SAA concentration of the same magnitude of the primary infestation peak, but within $24 \mathrm{~h}$ postsecondary infestation. Within 2 weeks of infestation, however, SAA levels had fallen to pre-infestation levels $(2.26 \pm 2.46 \mu \mathrm{g} / \mathrm{mL})$ and they remained at this level until the end of the trial.

\section{Analysis of serum IgG levels to the mite antigen Pso o 2} By week 2 post-primary infestation (PIT trial) the mean normalised $\mathrm{OD}_{450}$ value measured in ELISAs to detect Pso o 2-specific IgG in sheep serum had increased from $0.57 \pm 0.02$ pre-infestation to $1.197 \pm 0.115(p \leq 0.05)$ and continued to increase until week 6 post-primary infestation (Figure 5c). The antigen-specific IgG levels increased, exponentially, over time during the primary infestation period up to the point of treatment by 0.034 $\mathrm{OD}_{450}$ units per day $(95 \% \mathrm{CI}=0.032-0.037)$. By week 3 post-infestation, 9 out of 12 animals showed an "elevated" Pso o 2-specific IgG level (Table 3). After termination of the primary infestation with endectocide, the mean $\mathrm{OD}_{450}$ decreased from a 2 weeks post-treatment value of $2.66 \pm 0.07$ to $1.73 \pm 0.10$ by 3 weeks post-treatment $(p \leq 0.001)$. After this initial decline, the $\mathrm{OD}_{450}$ values continued to decline slowly and did not return to preinfestation levels until the start of the secondary infestation trial, which was 14 weeks post-treatment. The half life of the antibody levels to Pso o 2 following endectocide treatment was estimated as being 56 days with a decay rate of 0.012 $\mathrm{OD}_{450}$ units per day $(95 \% \mathrm{CI}=0.011-0.014)$. More than half of the sheep in the PIT trial continued to show elevated Pso o 2-specific IgG levels at 14 weeks post-treatment. As the anti-Pso o 2 antibody levels increased at approximately three times the rate at which they decreased, it is evident that antibody levels were not an accurate measure of current disease status post-treatment, but were effective during the early diagnosis of sheep scab during this trial.

\section{Assessment of APP levels in sheep during a field outbreak of sheep scab}

The pre-infestation levels of $\mathrm{Hp}(0.23 \pm 0.22 \mathrm{mg} / \mathrm{mL})$ were comparable to those, pre-infestation, in the PIT experimental trial $(0.29 \pm 0.06 \mathrm{mg} / \mathrm{mL})$ (Figure 6a). Following a field-acquired infestation with $P$. ovis, serum $\mathrm{Hp}$ levels were statistically significantly elevated at the point of clinical diagnosis $(p \leq 0.01)$ but the decrease in Hp levels 2 months post-treatment was not statistically significant.

The pre-infestation levels of SAA $(1.07 \pm 0.24 \mu \mathrm{g} / \mathrm{mL})$ in these sheep (Figure $6 \mathrm{~b}$ ) were marginally higher than

Table 3 Distribution of sheep into "normal" and "elevated" groups for Pso o 2-specific serum IgG across the time course of a primary infestation with Psoroptes ovis and treatment with an endectocide (PIT trial)

\begin{tabular}{lcccccccccccccccccccccc}
\hline Week & $\mathbf{0}$ & $\mathbf{1}$ & $\mathbf{2}$ & $\mathbf{3}$ & $\mathbf{4}$ & $\mathbf{5}$ & $\mathbf{6}^{*}$ & $\mathbf{7}$ & $\mathbf{8}$ & $\mathbf{9}$ & $\mathbf{1 0}$ & $\mathbf{1 1}$ & $\mathbf{1 2}$ & $\mathbf{1 3}$ & $\mathbf{1 4}$ & $\mathbf{1 5}$ & $\mathbf{1 6}$ & $\mathbf{1 7}$ & $\mathbf{1 8}$ & $\mathbf{1 9}$ & $\mathbf{2 0}$ \\
\hline Normal & 12 & 9 & 4 & 3 & 1 & 1 & 0 & 0 & 0 & 0 & 0 & 0 & 0 & 1 & 1 & 1 & 2 & 4 & 5 & 5 & 6 \\
Elevated & 0 & 3 & 8 & 9 & 11 & 11 & 12 & 12 & 12 & 12 & 12 & 12 & 12 & 11 & 11 & 11 & 10 & 8 & 7 & 7 & 6 \\
\hline
\end{tabular}

* All animals were treated with injectable endectocide at week 6 post-infestation. In this analysis the animals were described as being "normal" as in day 0 (pre-infestation) or "elevated". As the results showed a normal distribution, all values above the 0.999 quantile of the distribution were considered to be elevated, giving a $99.9 \%$ specificity and undefined sensitivity. 


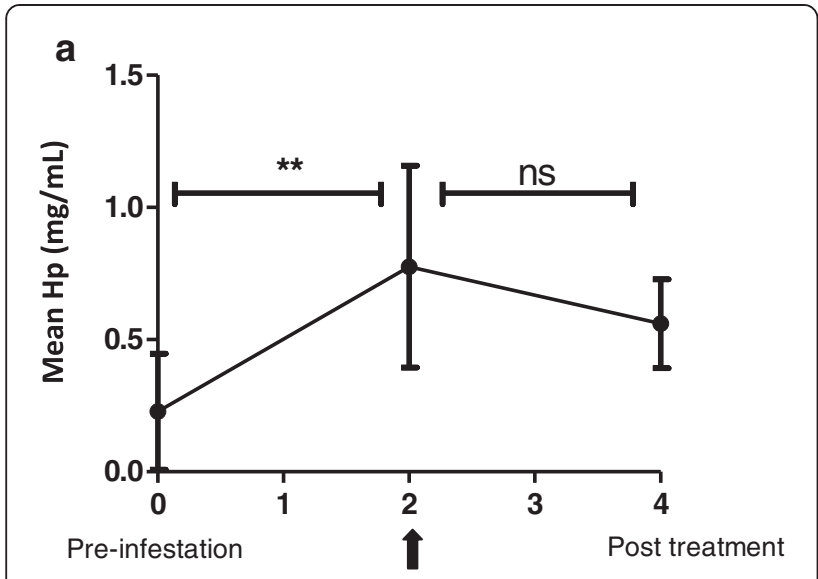

Diagnosed as clinically positive

Time (months)

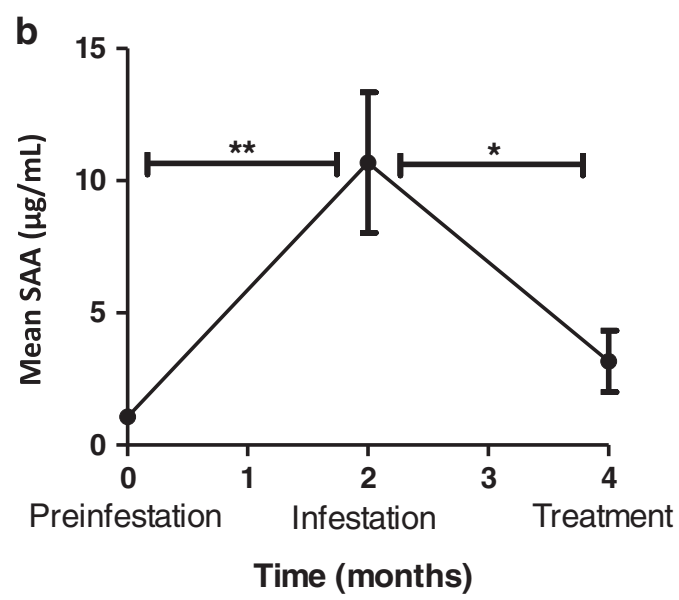

Figure 6 Quantitative analyses of serum acute phase proteins in sheep during a field-acquired infestation with Psoroptes ovis. Mean serum Hp (Panel a) and SAA (Panel b) concentrations ( \pm SE) from sheep $(n=12)$ pre-infestation, at the point of clinical diagnosis of sheep scab and 2 months post-treatment with an endectocide in a field outbreak of sheep scab. Hp measurement by commercial assay (ReactivLab) and SAA by commercial ELISA (Tridelta Development Ltd); sera from individual sheep diluted 1:50 for SAA assay and assayed in duplicate in both assays. ${ }^{* *} p \leq 0.01 ;{ }^{*} p \leq 0.05$ as determined by a one-way ANOVA (Graph Pad Prism).

those in the PIT experimental trial $(0.41 \pm 0.26 \mu \mathrm{g} / \mathrm{mL})$. At point of clinical diagnosis the recorded mean SAA $(10.69 \pm 2.66 \mu \mathrm{g} / \mathrm{mL})$ was statistically significantly higher than the pre-infestation level $(p \leq 0.01)$ and, unlike Hp, the decrease in SAA post-treatment was also statistically significant $(p \leq 0.05$, Figure $6 \mathrm{~b})$.

Hp and SAA levels during other common sheep infections and conditions

The serum Hp and SAA levels measured during most other common sheep infections were similar to those measured in sheep prior to infestation with $P$. ovis (Table 4).
Table 4 Serum Hp and SAA concentrations during common sheep diseases and conditions

\begin{tabular}{|c|c|c|}
\hline Infection/Condition & $\begin{array}{l}\text { Mean Hp } \\
(\mathrm{mg} / \mathrm{mL})\end{array}$ & $\begin{array}{c}\text { Mean SAA } \\
(\mu \mathrm{g} / \mathrm{mL})\end{array}$ \\
\hline Early gestation ${ }^{a}(n=10)$ & $0.33 \pm 0.01$ & $9.34 \pm 2.14$ \\
\hline Late gestation $^{\mathrm{b}}(n=10)$ & $0.52 \pm 0.08$ & $17.09 \pm 7.77$ \\
\hline $\begin{array}{c}\text { Gastro-intestinal } \\
\text { nematodes }^{c}(n=6)\end{array}$ & $0.04 \pm 0.03$ & $0.30 \pm 0.01$ \\
\hline $\begin{array}{l}\text { Liver Fluke (Fasciola } \\
\text { hepatica) }^{d}(n=6)\end{array}$ & $2.85 \pm 0.02$ & $0.22 \pm 0.01$ \\
\hline $\begin{array}{l}\text { Sucking lice (Linognathus spp. })^{e} \\
\qquad(n=4)\end{array}$ & $0.52 \pm 0.51$ & $68.47 \pm 22.33$ \\
\hline $\begin{array}{l}\text { Chewing lice (Bovicola ovis) } \\
\qquad(n=4)\end{array}$ & $0.65 \pm 0.31$ & $193.36 \pm 135.81$ \\
\hline $\operatorname{Orf}(n=6)^{f}$ & $0.21 \pm 0.01$ & $0.93 \pm 0.53$ \\
\hline Johnes disease ${ }^{9}(n=6)$ & $2.25 \pm 0.34$ & $21.43 \pm 10.47$ \\
\hline
\end{tabular}

$\mathrm{Hp}(\mathrm{mg} / \mathrm{mL} \pm \mathrm{SEM})$ measured by colorimetric assay (ReactivLab) and SAA ( $\mu \mathrm{g} /$ $\mathrm{mL} \pm \mathrm{SEM}$ ) assessed by SAA ELISA (Tridelta Development Ltd). All samples were assayed for individual animals in duplicate. "Early gestation was within 1 week of conception and blate gestation was 1 week prior to expected lambing date (samples from Moredun Research Institute (MRI)). 'Samples from sheep with field-acquired gastro-intestinal nematode (GIN) infections were from Firth Mains Farm, UK (MRI); species included Teladorsagia circumcincta and Nematodirus battus. ${ }^{d}$ Samples were taken from UK sheep with fieldacquired infections of liver fluke (samples provide by Dr H McDougall, MRI); e samples from lice infected but $P$. ovis-uninfested sheep (provided by Prof. $\mathrm{N}$. Sargison, R(D)SVS, UK; ${ }^{f}$ samples from sheep infected experimentally with orf virus or ${ }^{9}$ Mycobacterium avium subspecies paratuberculosis (MRI).

For example $\mathrm{Hp}$ and SAA levels during experimental gastrointestinal nematode infections were $0.04 \pm 0.03 \mathrm{mg} / \mathrm{mL}$ and $0.30 \pm 0.01 \mu \mathrm{g} / \mathrm{mL}$ respectively. The exceptions to this were during liver fluke infection and Johnes disease, where $\mathrm{Hp}$ levels were $2.85 \pm 0.02 \mathrm{mg} / \mathrm{mL}$ and $2.25 \pm 0.34 \mathrm{mg} / \mathrm{mL}$ respectively, which are of the same magnitude as those measured 4-5 weeks post-infestation with $P$. ovis. SAA levels during chewing and sucking lice infestations also showed the same magnitudes as 4-5 weeks postinfestation with $P$. ovis at $193.36 \pm 135.81 \mu \mathrm{g} / \mathrm{mL}$ and $68.47 \pm 22.33 \mu \mathrm{g} / \mathrm{mL}$ respectively.

\section{Discussion}

Here we identified, by proteomic and semi-quantitative immunoproteomic techniques, serum $\mathrm{Hp}$ and SAA as potential biomarkers for active sheep scab infestation. The qualitative results for SAA in the lesion resolving sheep (Figure 4a) where the band densities increased then decreased around 2 weeks before the lesion size changes, suggested that SAA levels in the serum of infested sheep may increase before obvious clinical signs were apparent, which would be an important factor in the pre-clinical diagnosis of sheep scab. However, when the levels of SAA and Hp in sera were quantified using assays, it was demonstrated that both APPs increased in serum concentration as $P$. ovis infestation progressed, but did not show statistically significant increases $(p \leq$ 0.001 ) until weeks $4-5$ post-infestation. Previous studies 
have described increases in serum $\mathrm{Hp}$ and SAA levels during a range of inflammatory diseases in ruminants $[16,23,24]$, but this is the first report of their elevation during sheep scab disease.

During a six week experimental infestation of sheep with $P$. ovis, Hp levels in serum increased by more than 10-fold to $3.5 \mathrm{mg} / \mathrm{mL}$ compared with pre-infestation levels, whereas SAA levels increased approximately 1000fold in the same period to $211 \mu \mathrm{g} / \mathrm{mL}$. These levels were similar to those of Hp and SAA measured in Alpine ibex (Capra ibex) infested with clinical sarcoptic mange (Sarcoptes scabiei infestation) where mean Hp levels during naturally-acquired infestation increased to $3.72 \pm$ $0.65 \mathrm{mg} / \mathrm{mL}$ compared with healthy, uninfested controls $(0.58 \pm 0.09 \mathrm{mg} / \mathrm{mL})$ and SAA levels were $130.7 \pm$ $0.16 \mu \mathrm{g} / \mathrm{mL}$ and $8.7 \pm 0.13 \mu \mathrm{g} / \mathrm{mL}$ in infested and uninfested animals respectively [25]. Generally, major APPs are present at undetectable or very low levels in the serum of healthy animals [26] but, recently, a study investigating the APP response to scrapie in sheep found large individual animal variation in $\mathrm{Hp}$ and SAA prior to clinical disease onset and it was suggested that this may be due to underlying subclinical conditions [27]. Previous work has indicated that $\mathrm{Hp}$ and SAA are non-specific in terms of inflammatory disease, injury or infection, but highly sensitive, effective markers of inflammation in ruminants $[16,24]$ suggesting that any inflammatory event in the animal may cause a temporary rise in $\mathrm{Hp}$ or SAA. In contrast to $\mathrm{Hp}$, serum SAA was measured at lower levels in sheep serum prior to $P$. ovis infestation; mean SAA concentration preinfestation during the PIT trial was $0.82 \pm 0.53 \mu \mathrm{g} / \mathrm{mL}$, representing only $0.29 \%$ of the peak SAA levels measured at week 5 post-infestation. This suggests that SAA may potentially be a more suitable $\mathrm{BM}$ than $\mathrm{Hp}$ to indicate current disease status during $P$. ovis infestation in sheep although the biochemical assay for $\mathrm{Hp}$ has practical advantages being an automated rapid assay which could be incorporated into routine biochemistry profile analysis in contrast to the ELISA system used for quantification of SAA.

In sheep infected with bacteria causing caseous lymphadenitis (CLA), serum Hp and SAA levels peaked by day 7 post-infection, which is considered to be the point at which the acute disease becomes a chronic infection [28]. Hp and SAA have also been previously reported to be measurable by day two post-infection in acute inflammatory diseases such as mastitis and metritis [16]. In contrast, the proportion of animals with a $P$. ovis-induced elevation in APPs did not occur until weeks 4-5 post-infestation in the case of $\mathrm{Hp}$ (Table 2), or marginally earlier at week 4 for SAA (Table 2). A possible explanation for this is that disease initiation in sheep scab has a "lag phase" at the start of infestation lasting for several weeks as the mites become established, after which the mite numbers increase exponentially [7]. Lesion size development follows this pattern in mite numbers, as the increasing numbers of mites move out from the margins of the lesion onto healthy skin, as shown by the lesion size data recorded in the TCT trial (Figure 1).

In order for a biomarker to be useful in the diagnosis of current disease status in sheep exposed to $P$. ovis, it is crucial that its levels in the serum decline rapidly after treatment or disease resolution. This study has demonstrated that $\mathrm{Hp}$ and SAA return to pre-infestation levels rapidly following termination of $P$. ovis infestation, with half-lives of 2.3 days for $\mathrm{Hp}$ and 0.84 days for SAA, illustrating the potential of including either or both of these APPs in an improved diagnostic test for sheep scab where knowledge of current disease status is important. For example, in the case of the Sheep Scab (Scotland) Order 2010, where confirmation of successful treatment is required before movement restrictions are lifted from the affected farm. The legislation allows lifting of movement restrictions to occur 16 days following successful treatment, when it is still difficult to assess by clinical examination. However, if $\mathrm{Hp}$ and /or SAA were used as BMs of current disease status, they would have returned to baseline levels before 16 days indicating successful treatment, as shown in Figure 5a and 5b.

SAA was a more accurate discriminatory indicator of current disease status in the PIT study than $\mathrm{Hp}$, due to the higher sensitivity and specificity obtained for the optimised cut-off values which were estimated using the data from the primary infestation and post-treatment element of the PIT trial.

At the cut-off levels established for SAA and $\mathrm{Hp}$ by statistical analysis of the PIT experimental trial results, it was evident that, during secondary experimental infestation (i.e. re-infestation), mean $\mathrm{Hp}$ levels in the sera of infested sheep were below the $\mathrm{Hp}$ cut-off point at all time points. In addition, for SAA, mean sera levels were above the cut-off point for 1 week only during this secondary infestation period. This may reflect the altered immunological landscape during the pathogenesis of a re-infestation, where lesion size and mite numbers are reduced compared with primary lesions [5] and may represent a limitation for the use of $\mathrm{BM}$ assays although field outbreak studies are now required to establish suitable cut-off values in practice.

When $\mathrm{Hp}$ and SAA levels were investigated during other common diseases and conditions of sheep, they were predominantly found to be at levels equivalent to sheep scab negative values, remaining below $0.6 \mathrm{mg} / \mathrm{mL}$ for $\mathrm{Hp}$ and $22 \mu \mathrm{g} / \mathrm{mL}$ for SAA. Importantly, as gastrointestinal nematodes (GIN) commonly affect sheep, Hp and SAA levels were not elevated during the GIN experimental infections tested here. Although $\mathrm{Hp}$ was 
elevated in liver fluke and Johnes disease and SAA was elevated in lice infestation in this analysis, it is evident from the standard errors in these results that there was large between animal variation and therefore these recorded measurements may be due to other underlying conditions. Analysis of additional sera samples from sheep infected with these diseases would therefore be required to confirm serum levels of $\mathrm{Hp}$ and SAA during these infections. In addition, as the BM assays react to other inflammatory conditions, they should be used in conjunction with the specific antibody assay which has been developed for sheep scab diagnosis and has been shown not to cross-react with other common sheep diseases and conditions, including biting lice [12]. Compared to Hp and SAA levels, the measurement of Pso o 2-specific IgG provided earlier, specific, diagnosis of $P$. ovis infestation as shown in Table 3 , where the majority of animals showed an elevated antigen-specific IgG response to Pso o 2 in the primary infestation by week 2 post-infestation. This was a similar response to that previously reported using this assay [12]. While this assay therefore provides rapid, sensitive and specific diagnosis of $P$. ovis infestation, antibody persistence (Figure 5c and [13]) prevents accurate diagnosis of current disease status e.g. after treatment or on disease resolution. When the decreases in $\mathrm{Hp}$ and SAA levels post-treatment were compared to those of the Pso o 2 specific IgG levels, it was evident that $\mathrm{Hp}$ and SAA gave a rapid indication of current disease status post-treatment, whereas high Pso o 2-specific IgG levels were still measurable 20 weeks post-treatment. The differences in half life also illustrated the differing responses to treatment i.e. 56 days for the Pso o 2-specific IgG response, compared to 2.3 days for $\mathrm{Hp}$ and 0.84 days for SAA. However, as serum Hp and SAA levels increase in many inflammatory diseases in ruminants [16] these APPs could not be used in isolation for the diagnosis of sheep scab. The relative merits of the tests measuring the Pso o 2-specific IgG response and APP levels indicate that a combined diagnostic test incorporating both elements would result in a highly specific test which would indicate both pathogen specific early infestation (Pso o 2-specific IgG levels) and current disease status after treatment (SAA and/or Hp levels). This would provide the sheep industry with a powerful diagnostic tool for sheep scab control or eradication schemes.

\section{Competing interests}

The authors declare they have no competing interests. PDE was a founding Director of ReactivLab Ltd and remains a shareholder and consultant.

\section{Authors' contributions}

Experimental design and planning: BW, AJN, STGB; Biomarker analyses: BW, EM, PDE; Statistical analyses BW, STGB, Gl; Drafting of the manuscript: BW, AJN, STGB, PDE, Gl. All authors read and approved the manuscript.

\section{Acknowledgements}

The authors gratefully acknowledge funding from a QMS/EBLEX/HCC postgraduate studentship to support the work of BW; STGB gratefully acknowledges UK Government funding from the Department for Environment, Food and Rural Affairs and AJN is funded by Scottish Government. GTI is partially funded by the Scottish Government. The authors would like to acknowledge Neil Inglis of the Proteomics group at MRI for performing the MALDI analysis and Mary Waterson, University of Glasgow for running the Hp assays.

\section{Author details}

${ }^{1}$ Moredun Research Institute, Pentlands Science Park, Midlothian EH26 OPZ, UK. 2Biomathematics \& Statistics Scotland (BioSS), King's Buildings, Mayfield Road, Edinburgh EH9 3JZ, UK. ${ }^{3}$ University of Glasgow, Sir Graeme Davies Building, 120 University Place, Glasgow G12 8TA, UK. ${ }^{4}$ Reactivlab Ltd, Garscube Estate, Bearsden Rd, Glasgow G61 1QH, UK

Received: 10 April 2013 Accepted: 17 October 2013 Published: 31 October 2013

\section{References}

1. Kirkwood A: History, Biology and Control of Sheep Scab. Parasitol Today 1986, 2:302-307.

2. Van den Broek AH, Huntley JF: Sheep scab: the disease, pathogenesis and control. J Comp Pathol 2003, 128:79-91.

3. Bisdorff B, Milnes A, Wall R: Prevalence and regional distribution of scab, lice and blowfly strike in Great Britain. Vet Rec 2006, 158:749-752.

4. The Scottish government [http://www.legislation.gov.uk/ssi/2010/419/ contents/made]

5. Bates P: Differences between primary and secondary infestations with the sheep scab mite, Psoroptes ovis. Vet Rec 2000, 146:528-529.

6. Bates $P$ : The effective diagnosis of sheep scab: differential diagnosis, sero-diagnosis and pen-side tests. Gov Vet J 2009, 20:32-37.

7. Bates $P$ : The pathogenisis and aging of sheep scab lesions. State Vet $J$ 1997, 7:11-15.

8. Sargison ND, Scott PR, Penny CD, Pirie RS: Effect of an outbreak of sheep scab (Psoroptes ovis infestation) during mid-pregnancy on ewe body condition and lamb birthweight. Vet Rec 1995, 136:287-289.

9. Fisher WF: Precipitating antibodies in sheep infested with Psoroptes ovis the sheep scab mite. J Parasitol 1972, 58:1218-1219.

10. Wassall DA, Kirkwood AC, Bates PG, Sinclair IJ: Enzyme linked immunosorbent assay for the detection of antibodies to the sheep scab mite Psoroptes ovis. Res Vet Sci 1987, 43:34-35.

11. Ochs H, Lonneux J, Losson BJ, Deplazes P: Diagnosis of psoroptic sheep scab with an improved enzyme linked immunosorbent assay. Vet Parasitol 2001, 96:233-242.

12. Nunn FG, Burgess ST, Innocent $G$, Nisbet AJ, Bates P, Huntley JF: Development of a serodiagnostic test for sheep scab using recombinant protein Pso o 2. Mol Cell Probes 2011, 25:212-218.

13. Burgess ST, Innocent G, Nunn F, Frew D, Kenyon F, Nisbet AJ, Huntley JF: The use of a Psoroptes ovis serodiagnostic test for the analysis of a natural outbreak of sheep scab. Parasit Vectors 2012, 5:7.

14. Atkinson AJ, Colburn WA, DeGruttola VG, DeMets DL, Downing GJ, Hoth DF, Oates JA, Peck CC, Schooley RT, Spilker BA, Woodcock J, Zeger SL: Biomarkers and surrogate endpoints: preferred definitions and conceptual framework. Clin Pharmacol Ther 2001, 69:89-95.

15. Burgess ST, Frew D, Nunn F, Watkins CA, McNeilly TN, Nisbet AJ, Huntley JF: Transcriptomic analysis of the temporal host response to skin infestation with the ectoparasitic mite Psoroptes ovis. BMC Genomics 2010, 11:624

16. Eckersall PD, Bell R: Acute phase proteins: biomarkers of infection and inflammation in veterinary medicine. Vet J 2010, 185:23-27.

17. Dobryszycka W: Biological functions of haptoglobin-new pieces to an old puzzle. Eur J Clin Chem Clin Biochem 1997, 35:647-654.

18. Bannikov G, Hinds C, Rajala-Schultz P, Premanandan C, Rings D, Lakritz J: Serum haptoglobin-matrix metalloproteinase 9 (Hp-MMP 9) complex as a biomarker of systemic inflammation in cattle. Vet Immunol Immunopathol 2011, 139:41-49.

19. Eckersall P, Duthie S, Safi S, Moffatt D, Horadagoda NU, Doyle S, Parton R, Bennett $D$, Fitzpatrick J: An automated biochemical assay for haptoglobin: prevention of interference from albumin. Comp Haematol Int 1999, 9:117-124 
20. Crawford K, Gow A, Marques A, Yool D, Eckersal P, McCulloch E, Lynn K, Mellanby R, Warman S: Serum haptoglobin concentrations in dogs with liver disease. Vet Rec. in press.

21. R Core Team [http://www.r-project.org]

22. R-Forge. [https://r-forge.r-project.org/projects/lme4]

23. Heegaard PM, Godson DL, Toussaint MJ, Tjornehoj K, Larsen LE, Viuff B, Ronsholt $L$ : The acute phase response of haptoglobin and serum amyloid A $(S A A)$ in cattle undergoing experimental infection with bovine respiratory syncytial virus. Vet Immunol Immunopathol 2000, 77:151-159.

24. Petersen HH, Nielsen JP, Heegaard PM: Application of acute phase protein measurements in veterinary clinical chemistry. Vet Res 2004, 35:163-187.

25. Rahman MM, Lecchi C, Fraquelli C, Sartorelli P, Ceciliani F: Acute phase protein response in Alpine ibex with sarcoptic mange. Vet Parasitol 2010, 168:293-298.

26. Cray C, Zaias J, Altman NH: Acute phase response in animals: a review. Comp Med 2009, 59:517-526.

27. Meling S, Bardsen K, Ulvund MJ: Presence of an acute phase response in sheep with clinical classical scrapie. BMC Vet Res 2012, 8:113.

28. Eckersall PD, Lawson FP, Bence L, Waterston MM, Lang TL, Donachie W, Fontaine MC: Acute phase protein response in an experimental model of ovine caseous lymphadenitis. BMC Vet Res 2007, 3:35-41.

doi:10.1186/1297-9716-44-103

Cite this article as: Wells et al.: Two major ruminant acute phase proteins, haptoglobin and serum amyloid $\mathrm{A}$, as serum biomarkers during active sheep scab infestation. Veterinary Research 2013 44:103.

\section{Submit your next manuscript to BioMed Central and take full advantage of:}

- Convenient online submission

- Thorough peer review

- No space constraints or color figure charges

- Immediate publication on acceptance

- Inclusion in PubMed, CAS, Scopus and Google Scholar

- Research which is freely available for redistribution 